\title{
基于测地回归模型的颖面复原
}

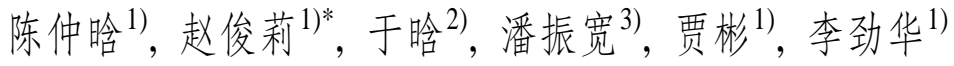 \\ ${ }^{1)}$ (青岛大学数据科学与软件工程学院 青岛 266071) \\ ${ }^{2)}$ (国防科技大学计算机学院 长沙 410073) \\ 3) (青岛大学计算机科学技术学院 青岛 266071) \\ (zhaojl@yeah.net)
}

\begin{abstract}
摘 要: 颖面复原是指根据一个未知颖骨的特征预测出对应的面貌, 在考古研究、医学整容、刑事案件调查等领域 有重要应用. 为解决㧫面复原过程中存在着数据量大、需要大量标定特征点的手工工作及颖面特征点定义困难的问 题, 针对三角网格表示的三维颖骨和面皮模型, 将颎面模型用从鼻尖出发的一组测地线表示, 提出了基于测地回归 的颅面复原方法. 该方法首先从三维人脸模型的鼻尖点提取一组测地线; 然后以颖骨和对应的人脸模型上提取的测 地线作为训练样本, 采用偏最小二乘回归统计模型复原未知颖骨对应人脸模型的测地线; 最后通过迭代最近点算法 将生成的测地线与人脸统计模型进行匹配, 实现复原测试颅骨的面貌. 在 192 套颅面数据库上进行了颅面复原实验, 将基于测地回归法复原的结果与主成分分析法和偏最小二乘回归法复原结果对比，实验结果表明，该方法能够提高 复原精度, 减少复原时间, 取得了良好的复原效果.
\end{abstract}

关键词: 㐿面复原; 测地线; 偏最小二乘回归; 测地回归统计模型

中图法分类号: TP391.41 DOI: 10.3724/SP.J.1089.2021.18320

\section{Craniofacial Reconstruction Based on Geodesic Regression Model}

\author{
Chen Zhonghan ${ }^{1)}$, Zhao Junli ${ }^{1)^{*}}$, Yu Han ${ }^{2)}$, Pan Zhenkuan ${ }^{3)}$, Jia bin ${ }^{1)}$, and Li Jinhua ${ }^{1)}$ \\ ${ }^{1)}$ (School of Data Science and Software Engineering, Qingdao University, Qingdao 266071) \\ ${ }^{2)}$ (College of Computer Science and Technology, National University of Defense Technology, Changsha 410073) \\ ${ }^{3)}$ (College of Computer Science and Technology, Qingdao University, Qingdao 266071)
}

\begin{abstract}
Craniofacial reconstruction refers to predicting the corresponding appearance based on the characteristics of an unknown skull. In order to solve the problem of large amount of data, manual work of calibrating feature points and difficulty in defining craniofacial feature points in the process of craniofacial reconstruction, for the three-dimensional skull and skin model represented by a triangular mesh, the craniofacial model is represented by a set of geodesics which are starting from the tip of a nose, thereby craniofacial reconstruction method based on geodesic regression is proposed. First, a set of geodesics is extracted from the nose tip point of the 3D face model; then the skull and the geodesics extracted from the corresponding face model are used as training samples, and the partial least square regression statistical model is used to reconstruct geodesics corresponding to the unknown skull; Finally, the iterative closest point algorithm is used to match the geodesics generated by the geodesic regression method with the facial statistical model to reconstruct the face of the test skull. Craniofacial
\end{abstract}

收稿日期：2020-04-16; 修回日期：2021-01-05. 基金项目：国家自然科学基金(61702293，61772294); 全国统计科学研究项目 (2020355); 山东省重点研发计划重大科技创新工程(2019JZZY020101). 陈仲晗(1992一), 男, 硕士研究生, 主要研究方向为计算机图 形学; 赵俊莉(1977一), 女, 博士, 副教授, 硕士生导师, CCF 会员, 论文通讯作者, 主要研究方向为计算机图形学、计算机视觉、虚 拟现实; 于晗(1990一), 男, 硕士, 主要研究方向为知识图谱、网络空间安全; 潘振宽(1966一), 男, 博士, 教授, 博士生导师, CCF 会 员, 主要研究方向为计算机视觉、图像处理; 贾彬(1998一), 男, 硕士研究生, 主要研究方向为计算机图形学; 李劲华(1963一), 男, 博 士, 教授, 硕士生导师, CCF 会员, 主要研究方向为软件工程. 
reconstruction experiments have been conducted on 192 sets of craniofacial data. The reconstruction results based on geodesic regression were compared with the reconstruction results of principal component analysis and partial least squares regression. The experimental results showed that our method can improve the reconstruction accuracy, reduce the reconstruction time, and achieve a good reconstruction effect.

Key words: craniofacial reconstruction; geodesic; partial least squares regression; geodesic regression statistical model

颅面复原技术 ${ }^{[1]}$ 是根据一个未知颅骨预测对 应面貌的技术，是近些年来的研究热点，在法医人 类学等领域具有重要意义. 在公安刑侦领域有助 于鉴别无名尸体身份，从而辅助破案. 在考古领域 可用于复原古人面貌，从而研究人类进化的历史; 在医学整容学方向也有助于预测整形面貌等. 1895 年解剖学家 His 和雕塑学家 Seffner 共同协作复原 出了著名音乐学家巴赫的面貌, 这标志着世界上 第一次采用科学的方法实现面貌复原. 随着计算 机水平的提高, 采用计算机辅助方法能更快速、客 观地实现颖面复原.

当前计算机辅助颅面复原法主要采用对颅骨 和面皮全部数据或手工提取的特征点实现㐿面复 原. 其中, 采用全部数据点进行复原计算量大; 而 选择特征点用于复原时, 特征点的选择对于统计 模型的表示能力具有重要的影响. 由于颅面形态 复杂, 生理特征点确定困难, 目前颅面特征点的选 择多采用手工标定的方式，不同的专家选定特征 点的个数和位置也不尽相同, 而且手动标记特征 点的方式费时费力且不准确, 因此如何自动地选 择用于统计复原模型的特征点是该方法的关键.

为解决计算机辅助颅面复原过程中存在的数 据计算量大、需要大量标定特征点的手工工作及颖 面特征点定义困难的问题，本文基于颉面内蕴的 几何结构, 提出了一种基于测地回归模型的颅面 复原方法, 将颅面模型用从鼻尖出发的一组测地 线表示，自动生成颖面几何特征点用于颅面复原 的回归模型中, 以复原出更高精度的未知颅骨的 面貌.

\section{1 研究现状}

早期, 颅面复原由有经验的画家和雕刻家采 用传统的手工复原方法完成 ${ }^{[2-3]}$, 复原效率低、精 度差，且由于不同专家掌握的形态学知识的不同， 复原面貌受专家的个人主观因素影响也不尽相同， 复原结果难以重现. 近年来, 采用计算机辅助的颅
面复原方法成为预测未知颖骨面貌的主流方法 ${ }^{[3]}$, 本文将相关工作分为 2 类方法介绍.

\section{1 基于知识的颖面复原方法}

基于知识的颖面复原方法包括对照匹配法和 模板变形法. 对照匹配法将参考硕面上的全套软 组织厚度数据全部加到待复原颖骨上来实现复原, 因为需要将参考枆面的软组织厚度一点点加到目 标颖骨上, 所以复原过程中消耗的时间长. 模板变 形法是通过未知颖骨的形状属性在数据库中寻找 一个相似的颖骨, 对此颖骨对应的面皮进行模板 变形完成复原. Vanezis 等 ${ }^{[4]}$ 提出了基于稀疏软组织 厚度的模板法, 首先在模板库中选择模板, 然后根 据软组织厚度数据将模板变形后贴合在待复原㐿 骨上，进而实现颅面复原. 该方法复原面貌效果更 接近原始面貌, 但五官等细节上的复原效果有待 提高. 税午阳等 ${ }^{[5]}$ 提出不同性别和年龄段的面部软 组织厚度不同，根据已知目标频骨的性别和年龄 等因素复原出面貌. 基于知识的颓面复原方法的 复原效果会受配准算法精度的影响, 复原中存在 较大误差.

\section{2 基于统计模型的颅面复原方法}

基于统计模型的颖面复原方法旨在通过对大 量的颖骨和面皮数据进行统计分析, 完成颖骨和面 皮间内在关系的建模，实现对未知颖骨面貌复原.

Claes 等 ${ }^{[6]}$ 将人脸采用面皮上稠密点表示, 颖 骨采用 52 个特征点表示, 然后建立统计形变模型; 再根据统计模型得出参数信息, 从而实现对未知 颅骨的面貌复原. 然而此类方法采用稀疏的表示 方法, 无法精确地描述复杂的颖骨信息. Claes 等 ${ }^{[7]}$ 还提出了完全自动地基于贝叶斯统计模型估计最 可能的人脸概率. $\mathrm{Hu}$ 等 ${ }^{[8]}$ 提出了一种融合全局和 局部的颖面复原方法, 构建了全局可变形模型, 以 眼睛、鼻子和嘴巴 3 个局部模型, 将全局和局部变 形模型与给定的头骨匹配, 并通过融合全局和局 部重建结果来获得重建的面部. 此类统计模型方 法虽然减少了人工的干预, 但其对颅骨和面部形 状相关性表述能力不足. 
当前，在统计模型中基于回归的统计模型可 以在回归模型中建立㐿骨和面皮之间的映射关系， 根据映射关系预测未知领骨的面貌，它能容易地 学习面皮和颖骨之间的本质关系. 基于回归的方 法包括基于线性回归和非线性回归的方法. Paysan 等 ${ }^{[9]}$ 提出了基于岭回归的方法，建立了颅骨和面皮 的关系，同时可以指定目标属性，使未知颅骨的复 原结果更加精确. Berar 等 ${ }^{[10]}$ 提出使用特征根回归 进行预测软组织曲面点的位置, 进而实现颖面复 原. Duan 等 ${ }^{[11]}$ 使用多线性子空间分析提取颎面子 空间特征，采用偏最小二乘回归 (partial least squares regression, PLSR)方法对颅骨和面皮进行 映射，更好地突出了面皮和颖骨之间的关系. Duan 等 ${ }^{[12]}$ 和 Huang 等 ${ }^{[13]}$ 则基于 PLSR 建立了颎骨和面 皮的关系，并对模型进行了分区回归复原，显著提 高了五官等复原的细节, 以及复原的精度. 该方法 不需要参数, 因此不会像岭回归一样受到参数的 影响. 通常在学习面皮和频骨关系之前, 由于不能 消除不同个体之间的区域位置不一致问题，因此 降低了颅面复原的准确性. 为了解决这个问题, Deng 等 ${ }^{[14]}$ 提出坐标调整和新的融合策略, 可以显 著改善颖面复原效果.

$\mathrm{Li}$ 等 ${ }^{[15]}$ 提出了一种基于非线性回归的最小二乘 支持向量回归(least squares support vector regression, LSSVR)方法复原未知颖骨面貌，它具有发现变量 之间非线性关系的灵活性，复原效果显著.

从上述研究可见, 回归模型能够更好地表示 颖骨决定面貌这一本质关系, 但复原过程中存在 着数据量大、需要大量标定特征点的手工工作及颖 面特征点定义困难的问题，本文将㐿面模型用从 鼻尖出发的一组测地线表示，自动生成颖面几何 特征, 结合 PLSR 统计模型提出了基于测地回归的 颖面复原方法.

\section{2 本文方法}

在对颖面内蕴几何特征进行分析的基础上, 本文提出基于测地回归模型的颖面复原方法, 从 面皮中提取 $m$ 条测地线, 将训练颖骨和对应面皮 的测地线用主成分分析(principal component analysis, PCA)方法 ${ }^{[16]}$ 进行降维; 然后将降维后的颖骨 和测地线数据送人 PLSR 模型中, 计算出测地线 和颖骨的映射关系, 并据此关系估计出未知颖骨 对应面貌的测地线; 最后再利用复原出的测地线 和人脸统计模型复原出面部. 该方法的流程如图 1 所示.

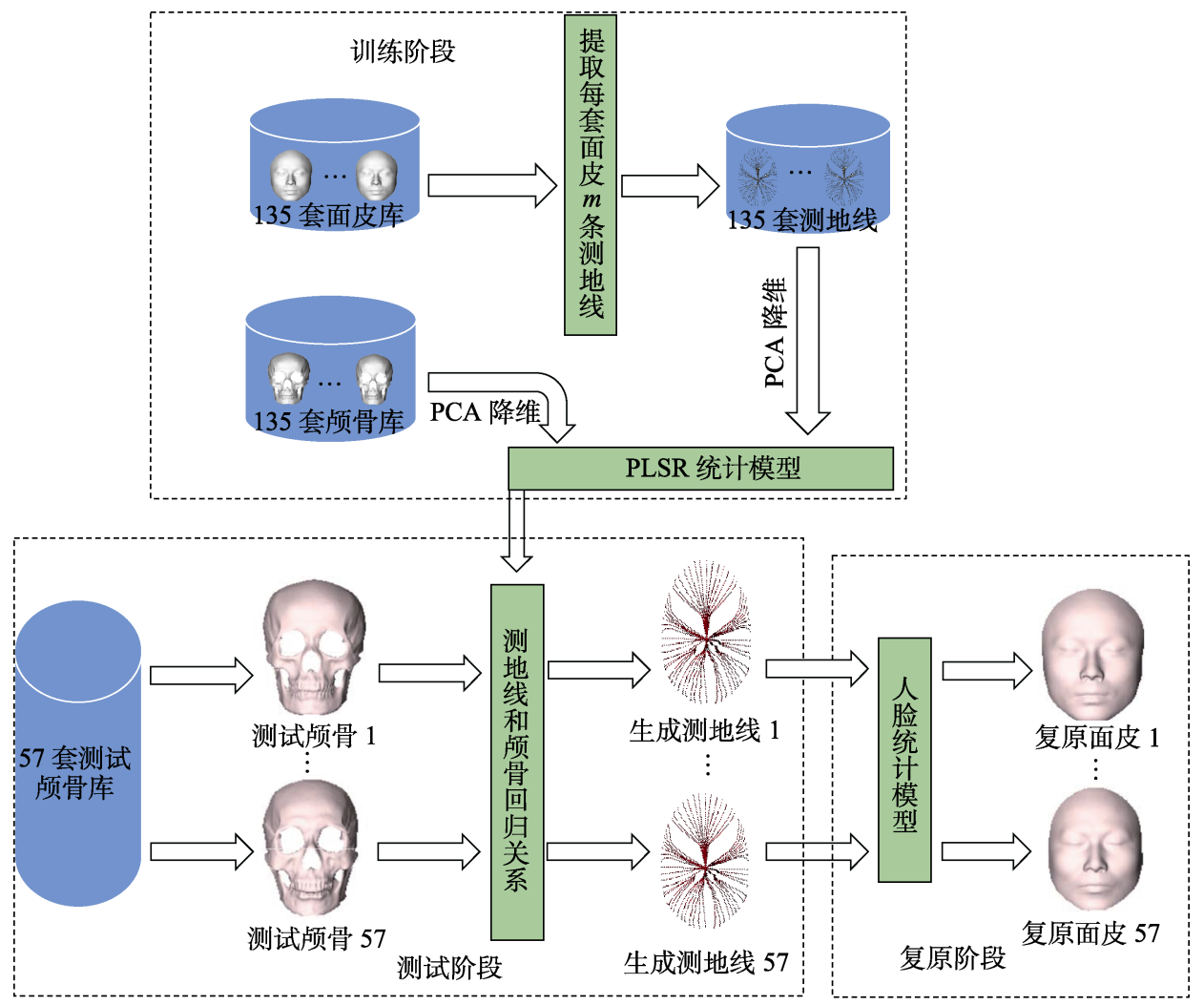

图 1 本文方法流程图 


\section{1 构建统一的三维硕面模型}

本文所使用的实验数据来自北京师范大学虚 拟现实应用教育部工程研究中心的颖面 $\mathrm{CT}$ 数据 库, 包括 192 套颖骨和面貌数据, 这些数据均由 CT 采集的颖面图像经过 Marching Cube 算法 ${ }^{[17]}$ 重 建得到三维颖骨和面貌模型. 为避免姿态、大小和 坐标系对实验生成结果的影响, 本文对所有的三维 㐿骨和面貌模型进行了统一坐标系 ${ }^{[18-19]}$ 、归一化 ${ }^{[20]}$ 和配准 ${ }^{[21-22]}$ 的预处理，具体方法如下:

(1) 建立统一的法兰克福坐标系

左侧耳门点 $p_{\mathrm{L}}$ 、右侧耳门点 $p_{\mathrm{R}}$ 和左侧眼 眶下缘点 $p_{\mathrm{M}} 3$ 点构成法兰克福平面, 再加上眉心 点 $p_{\mathrm{V}}$, 即可构建法兰克福坐标系：以 $p_{\mathrm{L}} p_{\mathrm{R}}$ 为法向 量且过眉心点 $p_{\mathrm{V}}$ 的平面与直线 $p_{\mathrm{L}} p_{\mathrm{R}}$ 的交点为法兰 克福坐标系的原点 $O, p_{\mathrm{L}}$ 到 $p_{\mathrm{R}}$ 的方向为 $X$ 正方向, 以法兰克福平面的法向为 $Z$ 轴正方向, 以右手法 则确定 $Y$ 轴, 最终在颖面上建立的法兰克福坐标 系如图 2 所示.
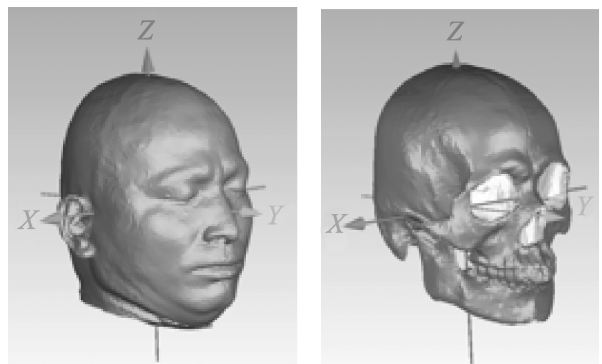

图 2 法兰克福坐标系下的颖面模型

(2) 统一姿态与数据归一化

建立好法兰克福坐标系后，将所有的颅骨原 始数据, 通过旋转变换到法兰克福坐标系, 即在统 一的坐标系下. 由于颖面大小不统一, 因此本文将 所有的颕骨和面貌模型数据都除以两耳之间的距 离, 即 $\left\|p_{\mathrm{L}}-p_{\mathrm{R}}\right\|$, 进行了归一化处理, 使得归一化 后的颉面数据尺度保持一致.

(3) 枆骨和面貌数据配准

在进行㐿骨和面貌数据配准时，首先选择一 套㐿骨和面皮模型作为参考。在研究颅面复原过 程中, 由于主要依据人脸的五官信息, 因此将参考 颅骨和面皮的后脑勺部分切除，保留前半部分，以 减少模型点数，提高实验运行速度. 然后使用非刚 性的薄板样条(thin plate spline, TPS) ${ }^{[23-24]}$ 算法数据 配准方法进行配准 ${ }^{[21-22,24]}$, 配准后㐿骨之间以及 面皮之间建立了对应关系. 如图 3 所示，每个面皮 包含 40969 个点, 每个顽骨包含 41059 个点, 具有 统一的姿态、相同的点数及语义.

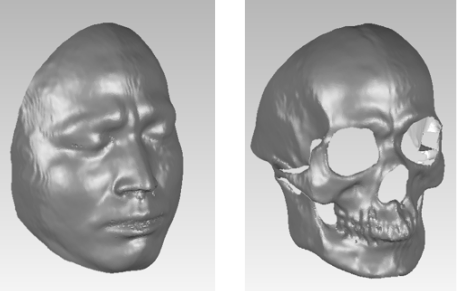

图 3 TPS 算法配准结果

\section{2 测地线的提取}

测地线 ${ }^{[25]}$ 是曲面上 2 点之间最短距离的路径, 是曲面固有的一个特征，具有内蕴性. 文献[26-27] 在三维人脸曲面上提取测地线的具体步骤如下:

(1) 定位测地线源点和终点

鼻尖点位于人脸的中间位置，也是整个面皮 中最突出的、最明显的部分. 从硕面中提取测地线 时, 首先在面皮中要找到鼻尖点的位置作为测地 线的源点. 在经过统一坐标系 ${ }^{[18-19]}$ 、归一化 ${ }^{[20]}$ 和配 准 ${ }^{[21-22]}$ 的预处理后得到统一姿态的三维㐿面模型 中, 找到模型上 $y$ 值最大的点, 即为鼻尖点, 将其 作为测地线的起点.

接下来将确定颖面边界上等分点作为测地线 的终点. 三维颖面模型由三角形网格表示, 在三维 颖面内部每条边都是 2 个三角形的公共边, 如果颖 面上的直线只属于一个三角形的边, 那么此边即 为三维㐿面边界上一条边, 从而能够确定颖面边 界. 确定了颖面的边界后, 采用人脸过鼻尖点的对 称面与人脸边界的交点作为提取第 1 条测地线的 目标点, 连接鼻尖点和第 1 个边界点作为提取面皮 的第 1 条测地(如图 4 所示). 然后在乑面的边界上 按相同的角度 $\alpha$ 确定 $m$ 个等分点 $B=\{b \mid \alpha \in A\}$. 其中, $A=\left\{0, \frac{2 \pi}{m}, \frac{4 \pi}{m}, \cdots, \frac{2 \pi(m-1)}{m}\right\}$, 将这些角度 等分点 $B$ 定义为测地线的目标点.

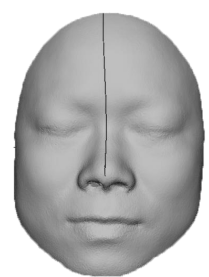

图 4 颉面第 1 条测地线

(2) 计算㐿面测地线

计算鼻尖点到边界等分点的测地线可以采用 测地线算法 ${ }^{[28-31]}$. 由于颖面复原需要计算大量样 本的多条测地线, 因而不同于文献[25-26], 本文选 取快速、鲁棒的 Fast marching 算法计算测地线. 
Fast marching 算法由 Sethian ${ }^{[32]}$ 于 1996 年提 出, Kimmel 等 ${ }^{[33]}$ 将该算法扩展到通用三角网格, 并提出了 Fast marching 方法的几何解释. 如图 5 所示, 计算 2 点之间的测地线即求顶点 $x_{0}$ 到 $x$ 之间 的最短线，其中 $d\left(x_{1}\right)$ 是源点 $x_{0}$ 到 $x_{1}$ 之间的最短路 径, $d\left(x_{2}\right)$ 是源点 $x_{0}$ 到 $x_{2}$ 之间的最短路径, 顶点 $x_{0}$ 到 $x$ 的距离最短路径 $d(x)$ 由三角面片 $\left(x, x_{1}, x_{2}\right)$ 与 $d\left(x_{1}\right), d\left(x_{2}\right)$ 更新求出, 路径可以穿过网格面片.

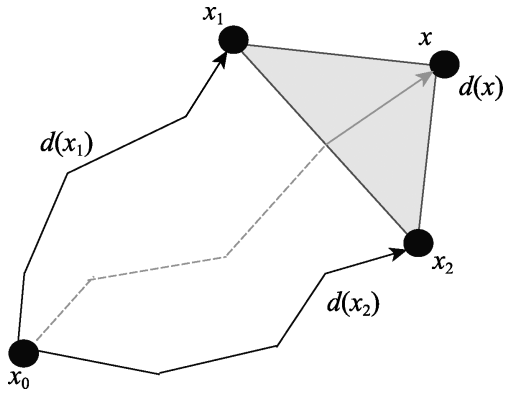

图 5 提取 $x_{0}$ 到 $x$ 的测地线

Fast marching 算法求解测地线时首先定位源 点 $x_{0}$, 其他顶点用 $x_{i}\left(x_{i} \in Q\right)$ 表示, $d\left(x_{i}\right)$ 表示距 离源点 $x_{0}$ 之间的距离, 即 $d\left(x_{0}\right)=0$, 其他顶点 $x_{i}$ 距离为 $d\left(x_{i}\right)=\infty$. 当 $Q$ 中存在点时, 从 $x_{0}$ 开始查 找与 $x_{0}$ 邻接位于 $Q$ 中的点 $x$, 依次查找在 $x$ 的相 邻顶点 $x^{\prime}$, 查找 $x^{\prime}$ 的一环邻域三角面片, 利用非 线性偏微分方程程函(Eikonal)方程计算顶点 $x^{\prime}$ 的 距离值 $d$, 并且记为 $d_{\text {min }}$, 更新顶点 $x^{\prime}$ 的距离值 $d\left(x^{\prime}\right)=\min \left\{d\left(x^{\prime}\right), d_{\text {min }}\right\}$. 当 $Q$ 中无顶点时, 返回 $d(x)$ 就是需要的最短路径. Fast marching 算法允许 测地线路径横穿多面体的面而不受限于边缘, 这 样可以保证提取的测地线最短.

依次计算鼻尖点到每个边界等分点的测地线, 可得到一组颖面测地线，用

$$
G=\left\{g_{\alpha} \mid \alpha \in A\right\}, A=\left\{0, \frac{2 \pi}{m}, \frac{4 \pi}{m}, \cdots, \frac{2 \pi(m-1)}{m}\right\}
$$

表示. 实验中取 $m=60$, 如图 6 所示提取 60 条测地 线. 为了计算准确的对应点, 对测地线进行了重采 样操作, 重采样的点数 $K=200$, 实验证明重采样 中的点数可以描述测地线的基本特征.

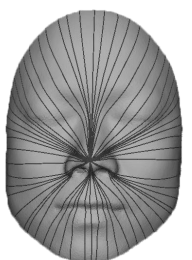

图 6 颅面测地线

\section{3 基于 PLSR 的测地回归模型}

\subsection{1 乑骨和面皮测地线数据降维}

统计学中的 PCA 方法属于经典的降维方法, 应用在众多科研领域. PCA 的作用就是将数据中的 重要信息提取出来，然后用正交主成分来表示，实 现对高维数据的降维.

对颅骨模型降维时，假设 $x_{1}, x_{2}, \cdots, x_{n}$ 为 $n$ 个 颅骨样本. 首先对 $n$ 个样本去均值, 即

$$
\boldsymbol{x}_{i}-\frac{1}{n} \sum_{j=1}^{n} \boldsymbol{x}_{j}=\boldsymbol{x}_{i}-\overline{\boldsymbol{x}}
$$

根据均值得出协方差矩阵

$$
\Sigma[\delta]=\frac{1}{n} E\left[\boldsymbol{X}^{\prime} \boldsymbol{X}^{\prime \mathrm{T}}\right]
$$

其中, $\boldsymbol{\Sigma}[\delta]$ 为数学期望; $\boldsymbol{X}^{\prime}$ 为乑骨样本去均值后 组成的矩阵. 求出协方差矩阵的特征值 $\left(\lambda_{1}, \cdots, \lambda_{n}\right)$ 和特征向量 $\left(\alpha_{1}, \cdots, \alpha_{n}\right)$. 贡献率 $\sum_{i=1}^{k} \lambda_{i} / \sum_{i=1}^{n} \lambda_{i}$, 其含 义为前 $k$ 个特征值所包含的信息.

$U_{x}$ 表示前 $k$ 个特征向量构成的矩阵，PCA 降 维后的颖骨为 $\boldsymbol{x}_{i}^{\prime}=\boldsymbol{U}_{x}^{\mathrm{T}}\left(\boldsymbol{x}_{i}-\overline{\boldsymbol{x}}\right)$. 同理, PCA 降维后 的面皮测地线为 $\boldsymbol{y}_{i}^{\prime}=\boldsymbol{U}_{y}^{\mathrm{T}}\left(\boldsymbol{y}_{i}-\bar{y}\right)$. 其中, $\boldsymbol{U}_{y}$ 是由面 皮测地线的协方差矩阵的特征向量组成的.

\subsection{2 基于 PLSR 测地回归模型复原颅面测地线}

PLSR 方法属于线性回归方法，可解决多自变 量和多因变量之间的关系, 具有预测功能, 在颖面 复原过程中，使用 PLSR 方法可以将颖骨和面皮测 地线之间的关系表示出来.

将乑骨训练样本构成的矩阵定义为 $\boldsymbol{X}=\left[\boldsymbol{x}_{1}\right.$, $\left.\cdots, x_{n}\right] \in \mathbb{R}^{p \times n}$, 面皮测地线的训练样本构成的矩 阵定义为 $\boldsymbol{Y}=\left[\boldsymbol{y}_{1}, \cdots, \boldsymbol{y}_{n}\right] \in \mathbb{R}^{q \times n}$. 从 $\boldsymbol{X}$ 和 $\boldsymbol{Y}$ 中分别 提取主成分

$$
\left\{\begin{array}{l}
\boldsymbol{t}_{1}=\boldsymbol{X} \boldsymbol{w}_{1} \\
\boldsymbol{u}_{1}=\boldsymbol{Y} \boldsymbol{c}_{1}
\end{array}\right.
$$

其中, $\boldsymbol{w}_{1}$ 为矩阵 $\boldsymbol{X}^{\mathrm{T}} \boldsymbol{Y} \boldsymbol{Y}^{\mathrm{T}} \boldsymbol{X}$ 的特征值中的最大值所 对应的特征向量, 根据特征向量可以得到颖骨样 本的首个主成分; $\boldsymbol{c}_{1}$ 为矩阵 $\boldsymbol{X}^{\mathrm{T}} \boldsymbol{Y} \boldsymbol{Y}^{\mathrm{T}} \boldsymbol{X}$ 的特征值中 的最大值所对应的特征向量, 同样可得出面皮样 本的首个主成分. 通过计算得到第 1 个主成分，进 而可以更新回归方程

$$
\left\{\begin{array}{l}
\boldsymbol{X}=\boldsymbol{t}_{1} \boldsymbol{d}_{1}^{\mathrm{T}}+\boldsymbol{X}_{1} \\
\boldsymbol{Y}=\boldsymbol{t}_{1} \boldsymbol{h}_{1}^{\mathrm{T}}+\boldsymbol{Y}_{1}
\end{array}\right.
$$

其中, $\boldsymbol{X}_{1}$ 和 $\boldsymbol{Y}_{1}$ 为首次回归后的残差矩阵; 回归系 数 $\boldsymbol{d}_{1}$ 和 $\boldsymbol{h}_{1}$ 的计算方法分别为 


$$
\left\{\begin{array}{l}
\boldsymbol{d}_{1}=\frac{\boldsymbol{X}^{\mathrm{T}} \boldsymbol{t}_{1}}{\left\|\boldsymbol{t}_{1}\right\|^{2}} \\
\boldsymbol{h}_{1}=\frac{\boldsymbol{Y}^{\mathrm{T}} \boldsymbol{t}_{1}}{\left\|\boldsymbol{t}_{1}\right\|^{2}}
\end{array}\right.
$$

采用同样的方式, 对 $\boldsymbol{X}_{1}$ 和 $\boldsymbol{Y}_{1}$ 提取主成分, 得 到 $\boldsymbol{t}_{2}=\boldsymbol{X}_{1} \boldsymbol{w}_{2}$ 和 $u_{2}=\boldsymbol{Y}_{1} \boldsymbol{c}_{2}$. 多次循环后可得到更多 的主成分, 最终得到的残差矩阵 $\boldsymbol{F}_{c}$ 极小, 可忽略 不计. 通过对

$$
\left\{\begin{array}{l}
\boldsymbol{X}=\boldsymbol{t}_{1} \boldsymbol{d}_{1}^{\mathrm{T}}+\boldsymbol{t}_{2} \boldsymbol{d}_{2}^{\mathrm{T}}+\cdots+\boldsymbol{t}_{c} \boldsymbol{d}_{c}^{\mathrm{T}} \\
\boldsymbol{Y}=\boldsymbol{t}_{1} \mathbf{h}_{1}^{\mathrm{T}}+\boldsymbol{t}_{2} \boldsymbol{h}_{2}^{\mathrm{T}}+\cdots+\boldsymbol{t}_{c} \boldsymbol{h}_{c}^{\mathrm{T}}+\boldsymbol{Y}_{c}
\end{array}\right.
$$

变形, 可得到测地线

$$
\boldsymbol{Y}=\boldsymbol{X} \boldsymbol{A}+\boldsymbol{Y}_{c}
$$

其中, $A=x \sum_{i=1}^{c} \boldsymbol{w}_{i} \boldsymbol{h}_{i}^{\mathrm{T}}$ 为回归系数. 参数 $c$ 的值可以 采用交叉有效性分析 ${ }^{[34]}$ 的方法确定. 设测试颖骨 在 PCA 子空间下的投影矩阵为 $\boldsymbol{x}_{t}^{\prime}$, 根据系数矩阵 $A$, 可得测试频骨对应的面皮测地线在 PCA 子空 间下的投影为 $\boldsymbol{y}_{\mathrm{tr}}^{\prime}=\boldsymbol{A} \boldsymbol{x}_{t}^{\prime}$, 再通过 $\boldsymbol{y}_{\mathrm{tr}}=\boldsymbol{U}_{y} \boldsymbol{y}_{\mathrm{tr}}^{\prime}+\bar{y}$ 复 原出未知颖骨的测地线.

\subsection{3 基于复原颖面测地线估计待复原颎面}

利用复原出的测地线估计出待复原颖面主要 分为 2 个步骤:

(1) 根据训练人脸数据建立人脸统计模型 $\boldsymbol{F}_{\text {model }}(\boldsymbol{\alpha})=\overline{\boldsymbol{F}}+\sum_{i=1}^{m} \boldsymbol{\alpha}_{i} \boldsymbol{h}_{i}$. 其中, $\overline{\boldsymbol{F}}=\frac{1}{N} \sum_{i=1}^{N} \boldsymbol{F}_{i}$ 是样本 向量的均值, 即平均脸; $\alpha=\left(\alpha_{1}, \cdots, \alpha_{m}\right)$ 是组合参 数, 且 $\boldsymbol{\alpha}_{i}$ 满足高斯分布; $\boldsymbol{h}_{i}$ 是原始样本向量的主 分量, 可以通过 $\boldsymbol{h}_{i}=\left(\boldsymbol{F}_{1}-\overline{\boldsymbol{F}}, \boldsymbol{F}_{2}-\overline{\boldsymbol{F}}, \ldots, \quad \boldsymbol{F}_{N}-\overline{\boldsymbol{F}}\right) \boldsymbol{U}_{i}$ 计算, $U_{i}$ 是第 $i$ 个特征值对应的特征向量. 在该人 脸统计模型中, 只要给定组合参数 $\boldsymbol{\alpha}$ 即可产生相 应的人脸 $\boldsymbol{F}_{\text {model }}(\alpha)$.

(2) 根据复原的测地线寻找合适的模型组合 参数, 使得由测地线复原出的颖面模型与人脸统计 模型的误差最小, 即最小化 min $\left\|\boldsymbol{F}_{\text {model }}(\boldsymbol{\alpha})-\boldsymbol{F}^{*}\right\|$. 其 中, $\boldsymbol{F}^{*}$ 是由测地线复原出的模型. 具体算法如下:

输人. 训练人脸数据 $\boldsymbol{F}_{i}=\left(x_{i 1}^{\mathrm{F}}, y_{i 1}^{\mathrm{F}}, z_{i 1}^{\mathrm{F}}, \cdots, x_{i n}^{\mathrm{F}}, y_{i n}^{\mathrm{F}}\right.$, $\left.z_{i n}^{\mathrm{F}}\right)(1 \leqslant i \leqslant N)$, 测试频骨复原出的测地线数据 $\boldsymbol{Y}$.

输出. 测试颖骨的人脸模型 $\boldsymbol{F}_{\text {model }}(\boldsymbol{\alpha})$.

Step1. 读人人脸数据, 计算人脸样本向量均值 $\overline{\boldsymbol{F}}=\frac{1}{N} \sum_{i=1}^{N} \boldsymbol{F}_{i}$.

Step2. 根据均值得出人脸样本向量协方差阵 $\boldsymbol{C}_{\mathrm{F}}=\frac{1}{N} \sum_{i=1}^{N}\left(\boldsymbol{F}_{i}-\overline{\boldsymbol{F}}\right)\left(\boldsymbol{F}_{i}-\overline{\boldsymbol{F}}\right)^{\mathrm{T}}$.
Step3. 根据 $\boldsymbol{C}_{\mathrm{F}}$ 计算人脸样本向量的特征值 $\boldsymbol{\lambda}_{i}$ 和对 应的特征向量 $\boldsymbol{U}_{i}(1 \leqslant i \leqslant m)$.

Step4. 计算人脸样本向量主分量 $\boldsymbol{h}_{1}=\left(\boldsymbol{F}_{1}-\overline{\boldsymbol{F}}\right.$, $\left.\boldsymbol{F}_{2}-\overline{\boldsymbol{F}}, \cdots, \boldsymbol{F}_{N}-\overline{\boldsymbol{F}}\right) \boldsymbol{U}_{i}(1 \leqslant i \leqslant m)$.

Step5. 建立人脸统计模型 $\boldsymbol{F}_{\text {model }}(\boldsymbol{\alpha})=\overline{\boldsymbol{F}}+\sum_{i=1}^{m} \boldsymbol{\alpha}_{i} \boldsymbol{h}_{i}$, 初 始设 $\boldsymbol{\alpha}=\mathbf{0}, \boldsymbol{F}_{\text {model }}(\boldsymbol{\alpha})=\overline{\boldsymbol{F}}$, 令 $\boldsymbol{F}^{*}$ 为测地线复原出的模型.

Step6. While $\left(\left\|\boldsymbol{F}_{\text {model }}(\boldsymbol{\alpha})-\boldsymbol{F}^{*}\right\|>\varepsilon\right)$ :

Step6.1. 使用迭代最近点(iterative closest point, ICP)方法确定人脸模型 $\boldsymbol{F}_{\text {model }}(\boldsymbol{\alpha})$ 与复原出的测地线 $\boldsymbol{Y}$ 的 对应点 $\boldsymbol{Y}^{\prime}$;

Step6.2. 令 $\boldsymbol{Y}^{\prime}-\boldsymbol{Y}=\boldsymbol{h}_{\boldsymbol{y}} \boldsymbol{\alpha}, \boldsymbol{h}_{\boldsymbol{y}}$ 是 $\boldsymbol{h}_{i}$ 中与测地线点 的对应分量, 利用 $\boldsymbol{\alpha}=\left(\boldsymbol{h}_{y}^{\mathrm{T}} \boldsymbol{h}_{y}\right)^{-1} \boldsymbol{h}_{y}^{\mathrm{T}}\left(\boldsymbol{Y}^{\prime}-\boldsymbol{Y}\right)$ 计算出人脸统 计模型的组合参数;

Step6.3. 根据 $\boldsymbol{\alpha}$ 更新人脸模型 $\boldsymbol{F}_{\text {model }}(\boldsymbol{\alpha})=\overline{\boldsymbol{F}}+$ $\sum_{i=1}^{m} \boldsymbol{\alpha}_{i} \boldsymbol{h}_{i}$, 并将 $\boldsymbol{F}^{*}=\boldsymbol{F}_{\text {model }}(\boldsymbol{\alpha})$.

Step7. End

Step8. Return $\boldsymbol{F}_{\text {model }}(\boldsymbol{\alpha})$.

\section{3 实验结果}

\section{1 不同方差贡献率的复原结果对比}

基于测地回归颖面复原方法，在训练模型阶 段的测地线特征值贡献率和颅骨特征值贡献率都 设置相同的值，生成的测地线对应的人脸模型分 别如表 1 所示, 不同贡献率对应的误差值如表 2 所 示. 可以看出, 当贡献率为 0.99 时, 复原颖面较好, 所以本文所有实验结果特征值贡献率均采用 0.99 .

表 1 不同样本特征值贡献率复原妙面对比

\begin{tabular}{lll}
\hline 样本贡献率 & 目标样本 \\
\hline 0.95 & 生成样本 \\
0.97 &
\end{tabular}

表 2 不同样本特征值贡献率复原硕面的误差

\begin{tabular}{ccccc}
\hline 贡献率 & 最小值 & 均值 & 最大值 & 方差 \\
\hline 0.95 & 0.0000015 & 0.0192704 & 0.0906121 & 0.0001791 \\
0.97 & 0.0000015 & 0.0192704 & 0.0906121 & 0.0001791 \\
0.99 & 0.0000002 & 0.0066950 & 0.0552015 & 0.0000230 \\
\hline
\end{tabular}




\section{2 测地回归颅面复原精度对比}

\subsection{1 不同测地线提取方法的复原精度对比}

本文采用 2 种不同的测地线提取方法对颖面 复原效果进行对比, 它们均以鼻尖点为测地线的 源点，但选择不同的测地线的边界点. 第 1 种提取 测地线方法是从颅面边界确定测地线边界等分点, 以颉面边界等分点为目标点提取的测地线, 如图 7 所示; 第 2 种提取测地线方法是以颎面边界点到鼻 尖点最近的距离定为等值距离，从㐿面上寻找所 有到鼻尖点的长度与等值距离相等的点组成边界 点, 按相同方法确定 60 个边界等分点, 进而如图 8 所示计算等值测地线. 然后结合 PLSR 方法进行㐿 面复原, 对 2 种方法分别从最小距离误差、平均距 离误差、最大距离误差和方差 4 个指标对比生成 颖面与原始颅面的精度. 误差值越小, 复原精度越 好, 从表 3 中的误差值可得, 第 1 种提取测地线方 法的复原结果误差更小，即选择第 1 种方法为本文 测地线提取方法.

\subsection{2 不同复原方法的精度对比}

本文使用了基于 PCA 的频面复原法、基于 $\mathrm{PLSR}^{[12]}$ 的颖面复原法和基于测地回归的㐿面复原 法进行颖面复原实验. 实验中共使用相同的 192 套 㐿面数据, 其中 135 套做训练, 57 套做测试, 将 3 种方法复原的颖面与原始面貌进行比较. 如表 4 所

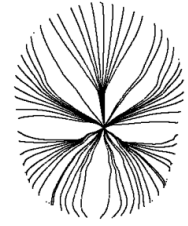

图 7 本文提取测地线

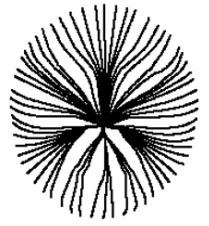

图 8 等值测地线
表 32 种方法生成颅面数据误差

\begin{tabular}{lcccc}
\hline \multicolumn{1}{c}{ 方法 } & 最小值 & 均值 & 最大值 & 方差 \\
\hline 等值测地回归 & 0.0000006 & 0.0153832 & 0.0671637 & 0.0001106 \\
测地回归 & 0.0000005 & 0.0113571 & 0.0506172 & 0.0001392 \\
\hline 误差 & 0.0000001 & 0.0040261 & 0.0165465 & 0.0000824 \\
\hline
\end{tabular}

表 43 种方法复原结果比较

\begin{tabular}{|c|c|c|c|c|c|c|}
\hline \multirow{2}{*}{ 目标面皮 } & \multicolumn{3}{|c|}{ 生成面皮 } & \multicolumn{3}{|c|}{ 生成误差 } \\
\hline & $\mathrm{PCA}^{[16]}$ & $\mathrm{PLSR}^{[12]}$ & 测地回归 & $\mathrm{PCA}^{[16]}$ & $\mathrm{PLSR}^{[12]}$ & 测地回归 \\
\hline
\end{tabular}


示, 生成误差中蓝色区域代表误差为零, 红色区域 代表误差最大. 通过表 4 中的生成面皮和生成误差 可以清晰地看出, 基于测地回归模型复原法复原 的面貌非常接近原始面貌. 结果表明, 基于测地回 归模型的颖面复原面貌误差最小，优于 PCA 和 PLSR 方法.

将 PCA，PLSR 方法生成的颖面误差与测地回 归方法生成的颎面误差进行对比，结果分别如表 5 和表 6 所示.

接下来将 PCA, PLSR 和测地回归方法 3 种方 法生成 57 套颖面与原始面貌之间的误差单独进行 对比. 图 9a 中, 因为基于 PLSR 和测地回归 2 种方 法的最小值比基于 PCA 方法的最小值小，所以无 法清晰地看出 PLSR 和测地回归方法的变化曲线. 从图 9b 误差平均值、图 9c 误差最大值和图 9d 方
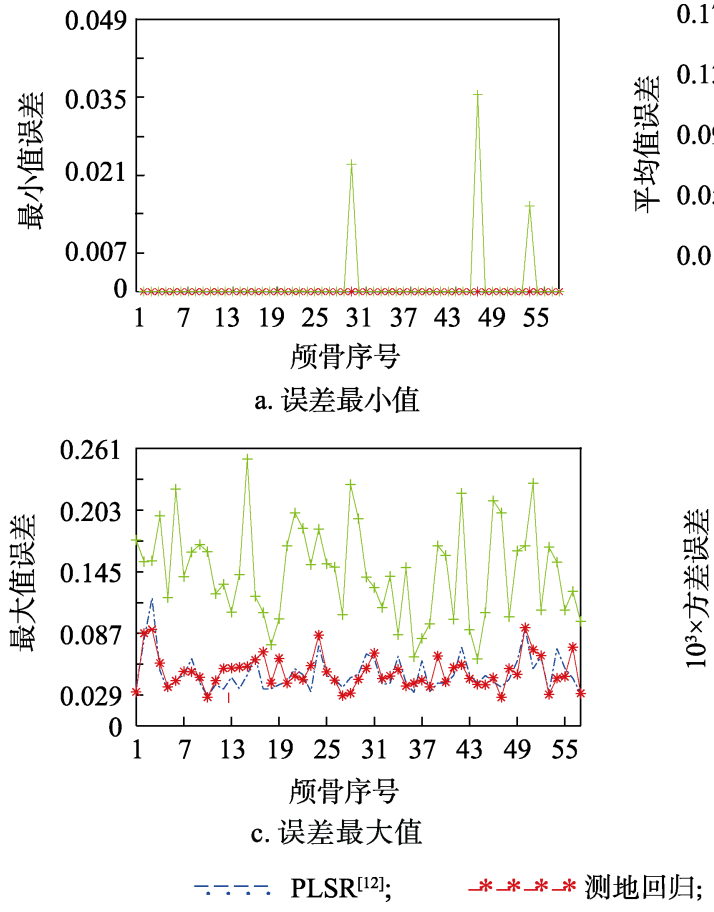

差误差的折线图可以看出，基于测地回归方法复 原㐿面的误差值曲线总体处于最低，而基于 PCA 方法复原颖面的误差值总体最高，说明基于测地回 归方法复原的㐿面的准确程度高于其他 2 种方法.

表 5 与 PCA 方法生成颅面数据误差对比

\begin{tabular}{lcccc}
\hline \multicolumn{1}{c}{ 方法 } & 最小值 & 均值 & 最大值 & 方差 \\
\hline PCA $^{[16]}$ & 0.0030868 & 0.0511509 & 0.1456021 & 0.0010308 \\
测地回归 & 0.0000005 & 0.0113571 & 0.0506172 & 0.0001392 \\
\hline 误差 & 0.0030863 & 0.0397938 & 0.0949849 & 0.0008916 \\
\hline
\end{tabular}

表 6 与 PLSR 方法生成颅面数据误差对比

\begin{tabular}{lcccc}
\hline \multicolumn{1}{c}{ 方法 } & 最小值 & 均值 & 最大值 & 方差 \\
\hline PLSR $^{[12]}$ & 0.0000005 & 0.0122614 & 0.0491077 & 0.0000741 \\
测地回归 & 0.0000005 & 0.0113571 & 0.0506172 & 0.0001392 \\
\hline 误差 & 0 & 0.0009043 & 0.0015095 & 0.0000651 \\
\hline
\end{tabular}

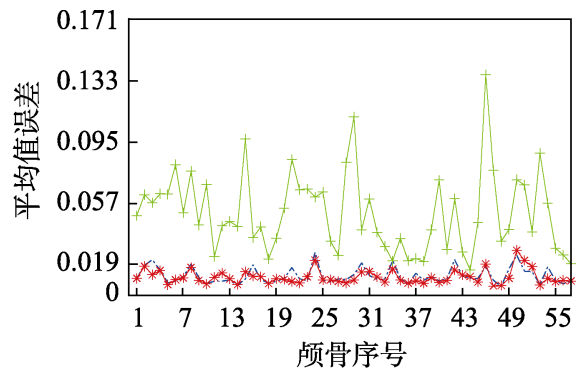

b. 误差平均值

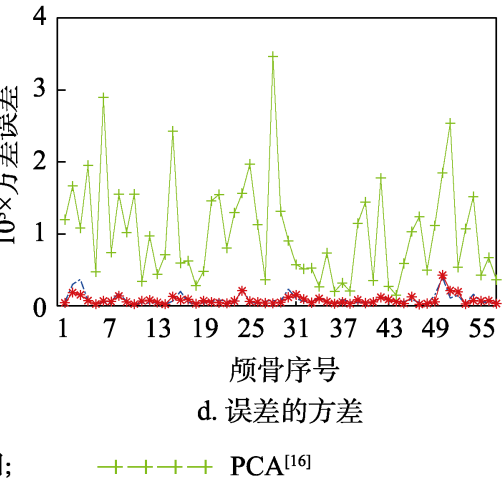

图 93 种方法复原颖面与原始面貌误差对比图

\section{3 测地回归颅面复原时间对比}

图 10 所示为对基于 PCA 方法、基于 PLSR 方 法和基于测地回归的复原方法复原人脸的测试时 间比较. 显然, 基于测地回归的方法用时最短, 复 原速度最快. 其原因主要是本文采用测地线上点 代替人脸上的所有点进行颖面复原，大大减少了 数据量, 由于使用更少的测地线上点进行颉面复 原，因而用本文方法复原颖面的速度更快.

综上，采用测地线表示颎面模型，基于测地回 归的颅面复原方法在复原时间和生成效果都优于 PLSR 和 PCA 方法.

\section{4 结 语}

本文提取三维人脸曲面上的测地线表示人脸, 自动定位颖面几何特征，结合 PLSR 回归构建了基 于测地回归的颖面复原模型. 以频面模型上提取 的测地线作为训练样本, 并使用 PLSR 统计模型复 原未知颅骨相应脸部模型的测地线. 最后, 通过 ICP 算法将采用测地回归方法生成的测地线与人 脸统计模型进行匹配, 实现的测地回归法复原的 面貌优于 PLSR 和 PCA 颅面复原的结果. 本文进行 的实验结果证明测地回归颅面复原方法可大大减 


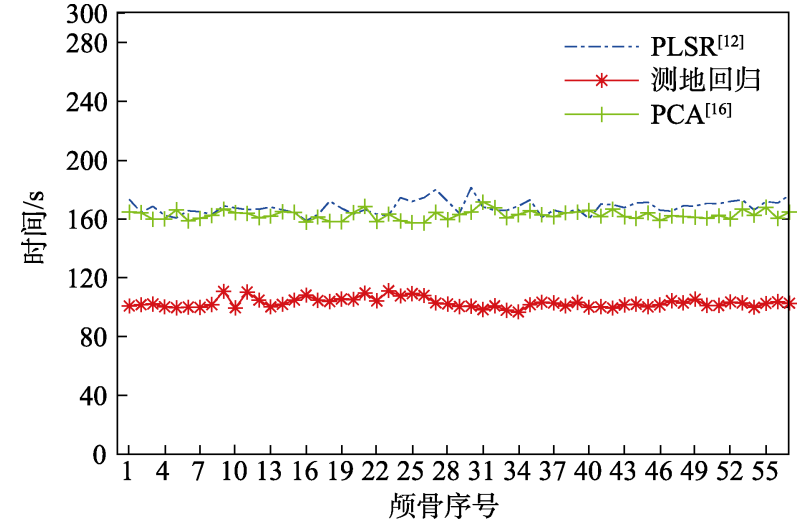

图 103 种方法颅面复原时间对比

少计算量，缩短颖面复原时间; 同时可以去除高维 颖面数据中的大量噪声数据, 自动提取颖面数据 的有效特征，提高复原精度. 本文采用的测地线表 示面皮的特征，仍属于人工提取面皮上特征的方 法, 接下来的研究借助更加强大的深度学习方法提 取面皮特征, 自动学习颖面特征, 实现颅面复原.

\section{参考文献(References):}

[1] Rinchon S, Arpita S, Mahipal S. 3D forensic facial reconstruction: a review of the traditional sculpting methods and recent computerised developments[J]. International Journal of Forensic Sciences, 2018, 3(1): 000134

[2] Quilliam T A. M. M. Gerasimov: the face finder. translated from German by A. H. Brodrick. London: Hutchinson, 1971. 199 pp. 16 pls. £ 3.00[J]. Antiquity, 1971, 45(180): 309-310

[3] Prag J, Neave R. Making faces: using forensic and archaeological evidence[M]. London: The Trustees of the British Museum, 1997

[4] Vanezis P, Vanezis M, McCombe G, et al. Facial reconstruction using 3-D computer graphics[J]. Forensic Science International, 2000, 108(2): 81-95

[5] Shui Wuyang, Zhou Mingquan, Ji Yuan, et al. Facial soft tissue thickness measurement and its application in craniofacial reconstruction[J]. Acta Anthropologica Sinica, 2013, 32(3): 345-353(in Chinese)

(税午阳, 周明全, 纪元, 等. 面部软组织厚度测量及其在面 貌复原中的应用 [J]. 人类学学报, 2013, 32(3): 345-353)

[6] Claes P, Vandermeulen D, de Greef S, et al. Computerized craniofacial reconstruction: conceptual framework and review[J]. Forensic Science International, 2010, 201(1-3): 138-145

[7] Claes P, Vandermeulen D, de Greef S, et al. Bayesian estimation of optimal craniofacial reconstructions[J]. Forensic Science International, 2010, 201(1-3): 146-152

[8] Hu Y L, Duan F Q, Zhou M Q, et al. Craniofacial reconstruction based on a hierarchical dense deformable model[J]. EURASIP Journal on Advances in Signal Processing, 2012, 2012(1): Article No.217

[9] Paysan P , Lüthi M, Albrecht T, et al. Face reconstruction from skull shapes and physical attributes[C] //Proceedings of the 31st DAGM Symposium. Heidelberg: Springer, 2009: 261-270

[10] Berar M, Tilotta F M, Glaunès J A, et al. Craniofacial reconstruction as a prediction problem using a Latent Root Regression model[J]. Forensic Science International, 2011, 210(1-3): 228-236

[11] Duan F Q, Yang S, Huang D H, et al. Craniofacial reconstruction based on multi-linear subspace analysis[J]. Multimedia Tools and Applications, 2014, 73(2): 809-823

[12] Duan F Q, Huang D H, Tian Y, et al. 3D face reconstruction from skull by regression modeling in shape parameter spaces[J]. Neurocomputing, 2015, 151(part 2): 674-682

[13] Huang D H, Duan F Q, Deng Q Q, et al. Face reconstruction from skull based on partial least squares regression[C] // Proceedings of the 7th International Conference on Computational Intelligence and Security. Los Alamitos: IEEE Computer Society Press, 2011: 1118-1121

[14] Deng Q Q, Zhou M Q, Wu Z K, et al. A regional method for craniofacial reconstruction based on coordinate adjustments and a new fusion strategy[J]. Forensic Science International, 2016, 259: 19-31

[15] Li Y, Chang L, Qiao X J, et al. Craniofacial reconstruction based on least square support vector regression[C] // Proceedings of the IEEE International Conference on Systems, Man and Cybernetics. Los Alamitos: IEEE Computer Society Press, 2014: 1147-1151

[16] Berar M, Desvignes M, Bailly G, et al. 3D semi-landmarksbased statistical face reconstruction[J]. Journal of Computing and Information Technology, 2006, 14(1): 31-43

[17] Lorensen W E, Cline H E. Marching cubes: a high resolution 3D surface construction algorithm[J]. ACM SIGGRAPH Computer Graphics, 1987, 21(4): 163-169

[18] Duan F Q, Yan Y C, Li Y, et al. Skull identification via correlation measure between skull and face shape[J]. IEEE Transactions on Information Forensics and Security, 2014, 9(8): 1322-1332

[19] Zheng X, Zhao J L, Lv Z H, et al. Skull similarity comparison based on SPCA[J]. Multimedia Tools and Applications, 2020, 79: 22423-22446

[20] Hu Y L, Duan F Q, Yin B C, et al. A hierarchical dense deformable model for 3D face reconstruction from skull[J]. Multimedia Tools and Applications, 2013, 64(2): 345-364

[21] Huang R K, Zhao J L, Duan F Q, et al. Automatic craniofacial registration based on radial curves[J]. Computers \& Graphics, 2019, 82: 264-274

[22] Zhao J L, Qi X, Wen C F, et al. Automatic and robust skull registration based on discrete uniformization[C] //Proceedings of the IEEE/CVF International Conference on Computer Vision. Los Alamitos: IEEE Computer Society Press, 2019: 431-440

[23] Brown B J, Rusinkiewicz S. Non-rigid range-scan alignment using thin-plate splines[C] //Proceedings of the 2nd International Symposium on 3D Data Processing, Visualization and Transmission. Los Alamitos: IEEE Computer Society Press, 2004: 759-765

[24] Bookstein F L. Principal warps: thin-plate splines and the decomposition of deformations[J]. IEEE Transactions on Pattern Analysis and Machine Intelligence, 1989, 11(6): 567-585 
[25] Du Peilin, Tu Changhe, Wang Wenping. Computing geodesics on point clouds[J]. Journal of Computer-Aided Design \& Computer Graphics, 2006, 18(3): 438-442(in Chinese) (杜培林, 屠长河, 王文平. 点云模型上测地线的计算 $[\mathrm{J}]$. 计 算机辅助设计与图形学学报, 2006, 18(3): 438-442)

[26] Zhao Junli, Wu Zhongke, Liu Cuiting, et al. 3D facial similarity comparison in shape space[J]. Optics and Precision Engineering, 2015, 23(4): 1138-1145(in Chinese)

(赵俊莉, 武仲科, 刘翠婷, 等. 形状空间下的 3D 面貌相似 性比较[J]. 光学精密工程, 2015, 23(4): 1138-1145)

[27] Zhao J L, Wu Z K, Pan Z K, et al. 3D face similarity measure by Fréchet distances of geodesics[J]. Journal of Computer Science and Technology, 2018, 33: 207-222

[28] Mitchell J S B, Mount D M, Papadimitriou C H. The discrete geodesic problem[J]. SIAM Journal on Computing, 1987, 16(4): 647-668

[29] Xin S Q, Wang G J. Improving Chen and Han's algorithm on the discrete geodesic problem[J]. ACM Transactions on Graph- ics, 2009, 28(4): 1-8

[30] Ying X, Huang C B, Fu X Z, et al. Parallelizing discrete geodesic algorithms with perfect efficiency[J]. Computer-Aided Design, 2019, 115: 161-171

[31] Wang X N, Fang Z, Wu J J, et al. Discrete geodesic graph (DGG) for computing geodesic distances on polyhedral surfaces[J]. Computer Aided Geometric Design, 2017, 52/53: 262-284

[32] Sethian J A. A fast marching level set method for monotonically advancing fronts[J]. Proceedings of the National Academy of Sciences of the United States of America, 1996, 93(4): 1591-1595

[33] Kimmel R, Sethian J A. Computing geodesic paths on manifolds[J]. Proceedings of the National Academy of Sciences of the United States of America, 1998, 95(15): 8431-8435

[34] Messick N J, Kalivas J H, Lang P M. Selecting factors for partial least squares[J]. Microchemical Journal, 1997, 55(2): 200-207 